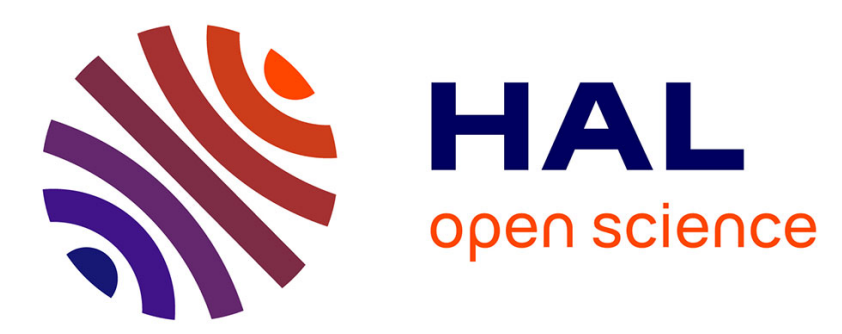

\title{
A class of nonlinear differential equations of second order in time
}

\author{
Michelle Schatzman
}

\section{To cite this version:}

Michelle Schatzman. A class of nonlinear differential equations of second order in time. Nonlinear Analysis: Theory, Methods and Applications, 1978, 2 (3), pp.355-373. 10.1016/0362-546X(78)900226. hal-01294058

\section{HAL Id: hal-01294058 \\ https://hal.science/hal-01294058}

Submitted on 26 Mar 2016

HAL is a multi-disciplinary open access archive for the deposit and dissemination of scientific research documents, whether they are published or not. The documents may come from teaching and research institutions in France or abroad, or from public or private research centers.
L'archive ouverte pluridisciplinaire HAL, est destinée au dépôt et à la diffusion de documents scientifiques de niveau recherche, publiés ou non, émanant des établissements d'enseignement et de recherche français ou étrangers, des laboratoires publics ou privés.

\section{다(1)(2)}

Distributed under a Creative Commons Attribution - ShareAlikel 4.0 International 


\title{
A CLASS OF NONLINEAR DIFFERENTIAL EQUATIONS OF SECOND ORDER IN TIME
}

\author{
Michelle Schatzman \\ Analyse Numerique t55-65, Universite P. et M. Curie, 4pl Jussieu, 75230 Paris Cedex 05, France
}

Key words: Existence and uniqueness, nonlinear equations of second order, monotone operators.

\section{INTRODUCTION}

IN WHAT follows, $\phi$ will denote a lower semicontinuous convex proper function from $\mathbf{R}^{n}=H$ to $\mathbf{R} \cup\{+\infty\}$. Here proper means that $\phi \not \equiv+\infty$. The effective domain of $\phi$ is the set dom $\phi=$ $\left\{x \in \mathbf{R}^{N} / \phi(x)<+\infty\right\}$. We shall suppose that the interior of dom $\phi$ in $\mathbf{R}^{N}$ is not empty, and $\phi \geqslant 0$. These two assumptions do not restrict the generality. The scalar product in $H$ is denoted by $(x, y)$.

Let us recall that the subdifferential of $\phi$ is a (multivalued) operator defined on

$$
D(\partial \phi)=\{x \in H: \exists z \in H \text { such that } \phi(x+y)-\phi(x) \geqslant(z, y) \text { for all } y \in H\}
$$

with values

$$
\partial \phi(x)=\{z \in H: \phi(x+y)-\phi(x) \geqslant(z, y), \forall y \in H\} .
$$

The set $\partial \phi(x)$ is closed convex in $H$ and $\partial \phi$ is a cyclically monotone operator, i.e. for all $n \in \mathbf{N}$, for all $n$-tuple $\left(x_{1}, \ldots, x_{n}\right)$ of elements of $D(\partial \phi)$, for all $y_{i} \in \partial \phi\left(x_{i}\right)(1 \leqslant i \leqslant n)$

$$
\left(y_{n}, x_{n}-x_{n-1}\right)+, \ldots+\left(y_{2}, x_{2}-x_{1}\right)+\left(y_{1}, x_{1}-x_{n}\right) \geqslant 0 \text {. }
$$

We define $J_{\lambda}=(I+\lambda \partial \phi)^{-1}$ on $H ; J_{\lambda}$ is a contraction, and $\partial \phi_{\lambda}=\left(I+J_{\lambda}\right) / \lambda$ is the Yosida approximation to $\partial \phi ; \partial \phi_{\lambda}$ is Lipschitzian, with Lipschitz constant $1 / \lambda$ and $\partial \phi_{\lambda}(x) \in \partial \phi\left(J_{\lambda} x\right)$. If $\phi_{\lambda}(x)=\inf \left\{|x-y|^{2} /(2 \lambda)+(\phi / 2)(y)\right\}, \partial \phi_{\lambda}$ is the subdifferential of $\phi_{\lambda}$. Explicitly, $\phi_{\lambda}(x)=$ $\phi\left(J_{\lambda} x\right)+\left|x+J_{\lambda} x\right|^{2} /(2 \lambda)$ and $\phi_{\lambda}(x) \leqslant \phi(x)$. Moreover, $\lim _{\lambda \rightarrow 0} \phi_{\lambda}(x)-\phi(x)$ if $x \in H$. The general references for these results are refs $[1,2]$.

Before we proceed to the existence theorem, it is of some interest to give an explicit example. It will allow the reader to notice the difficulties of the problem.

Take $H=\mathbf{R}, K=\mathbf{R}^{+}, \phi=\psi$ the indicator function of $K$, i.e.

Then

$$
\begin{array}{ll}
\phi(x)=+\infty & \text { if } x \notin K \\
\phi(x)=0 & \text { if } x \in K
\end{array}
$$

$$
\begin{array}{ll}
\partial \phi(x)=\{0\} & \text { if } x>0 \\
\partial \phi(x)=(-\infty ; 0] & \text { if } x=0 \\
\partial \phi(x)=\varnothing & \text { if } x<0 .
\end{array}
$$


Assume $u_{0}>0$; we shall seek a solution $u$, which is locally Lipschitzian in $t$. The interesting case occurs when $u_{1}<0$. If $t<u_{0} / u_{1}$, the only solution of

$$
\frac{\mathrm{d}^{2} u}{\mathrm{~d} t^{2}}+\partial \psi_{k}(u) \ni 0
$$

is $u(t)=x_{0}+t u_{1}$. We must have $u(t) \geqslant 0$, for all $t \geqslant 0$. It is easy to check that any $u$ of the form

$$
\begin{array}{ll}
u(t)=0 \quad \text { for } & t_{0}=-u_{0} / u_{1} \leqslant t \leqslant t_{1} \\
u(t)=v\left(t-t_{1}\right) & \text { for } \quad t \geqslant t_{1}
\end{array}
$$

with $v>0$ and $-u_{0} / u_{1} \leqslant t_{1} \leqslant \infty$ is a solution. (see the figure below). If we assume that the energy is conserved, then necessarily $u^{0}(t)=-u_{1}\left(t+u_{0} \mid u_{1}\right)$ for $t \geqslant-u_{0} / u_{1}$. There is an

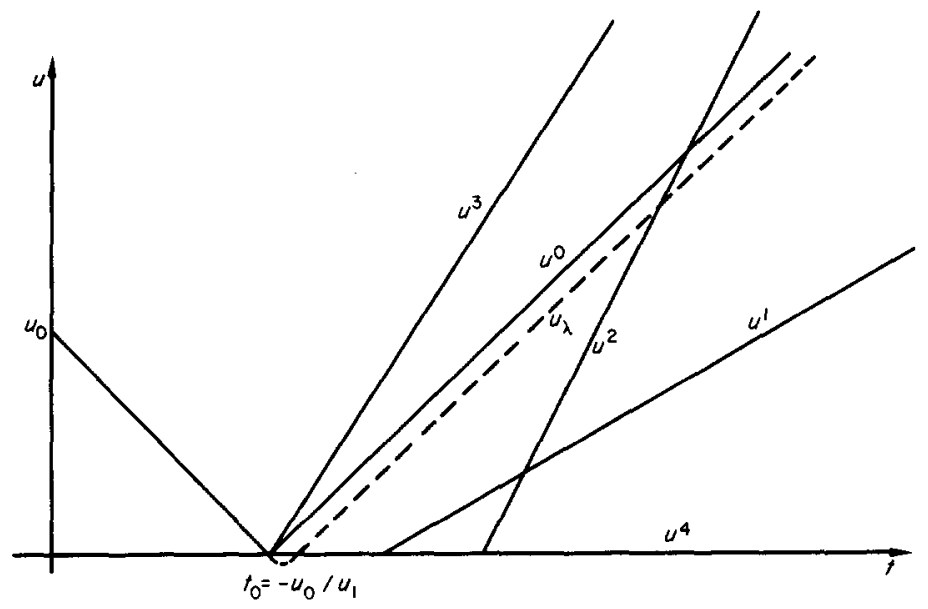

Fig. 1.

"optical reflection" in $-u_{0} / u_{1}$. The Yosida approximation gives, in the limit, the solution which conserves the energy. In fact, the approximating equation is

$$
\begin{array}{cc} 
& \frac{\mathrm{d}^{2} u_{\lambda}}{\mathrm{d} t^{2}}-\frac{u_{\lambda}^{-}}{\lambda}=0, \text { where } r^{-}=-\min (r, 0) ; \text { its solution is } \\
u_{\lambda}(t)=u_{0}+t u_{1} & 0 \leqslant t \leqslant-u_{0} / u_{1}=t_{0} \\
u_{\lambda}(t)=\sqrt{\lambda} u_{1} \sin \left[\left(t+t_{0}\right) / \sqrt{\lambda}\right] & t_{0} \leqslant t \leqslant t_{0}+\pi \sqrt{\lambda} \\
u_{\lambda}(t)=-u_{1}\left(t-\pi \sqrt{\lambda}-t_{0}\right) & t_{0}+\pi \sqrt{\lambda} \leqslant t .
\end{array}
$$

Clearly, the $u_{\lambda}$ converge to the energy conserving solution, $u^{0}$.

\section{THE EXISTENCE THEOREM}

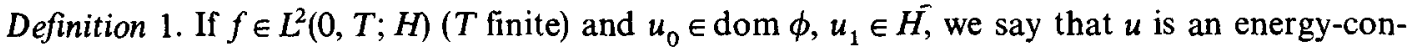
serving solution of the problem $(\mathrm{P})$ 


$$
\left(\mathrm{d}^{2} u / \mathrm{d} t^{2}\right)+\partial \phi(u) \ni f, \quad \text { "initial conditions" }
$$

when it satisfies the following requirements:

(1) $u \in W^{1, \infty}((0, T) ; H)$

(2) $u(t) \in \operatorname{dom} \phi, \forall t \in[0, T]$

(3) there exists a bounded measure $\mu$ with values in $H$, such that

$$
\left(\mathrm{d}^{2} u / \mathrm{d} t^{2}\right)+\mu=f \text { in the sense of distributions, }
$$

and

(4) for any continuous $v$, with values in $H$, such that $\phi(v) \in L^{1}(0, T)$, we have:

$$
\int_{0}^{T}(\phi(v)-\phi(u)) \mathrm{d} t \geqslant\langle\mu, v-u\rangle
$$

(5) $\mathrm{d} u / \mathrm{d} t$ has left and right limits for any $t \in[0, T]$ (with the necessary modifications at 0 and $T$ )

(6) energy is conserved:

$$
\left|\frac{\mathrm{d}^{+} u}{\mathrm{~d} t}(t)\right|^{2}+\phi(u(t))=\left|\frac{\mathrm{d}^{-} u}{\mathrm{~d} t}(t)\right|^{2}+\phi(u(t))=\left|u_{1}\right|^{2}+\phi\left(u_{0}\right)+\int_{0}^{t}(\dot{u}(s), f(s)) \mathrm{d} s
$$

almost everywhere on $[0, T]$; here $\dot{u}$ stands for $\mathrm{d} u / \mathrm{d} t$

(7) the initial conditions are satisfied in the following sense:

$$
u(0)=u_{0}
$$

and if $K_{0}$ is the closure of dom $\phi, \psi_{K_{0}}$ the indicator function of $K_{0}$ :

$$
-u_{1}+\frac{\mathrm{d}^{+} u}{\mathrm{~d} t}(0)+\partial \psi_{K_{0}}\left(u_{0}\right) \ni 0
$$

Theorem 1. (a) For any $f \in L^{2}(0, T ; H), u_{0} \in \operatorname{dom} \phi$ and $u_{1} \in H$, the problem (P) has an energyconserving. solution in the sense of Definition 1. This solution is obtained as the strong limit in $H^{1}((0, T) ; H)$ and the weak * limit in $W^{1, \infty}((0, T) ; H)$ of a subsequence of the sequence of solutions of $\left(P_{\lambda}\right)$ :

$$
\frac{\mathrm{d}^{2} u_{\lambda}}{\mathrm{d} t^{2}}+\partial \phi_{\lambda}\left(u_{\lambda}\right)=0, \quad u_{\lambda}(0)=u_{0}, \quad \frac{\mathrm{d} u_{\lambda}}{\mathrm{d} t}(0)=u_{1} .
$$

(b) Moreover, if $\phi$ is Lipschitzian in a neighbourhood of $u_{0}$ with respect to $K_{0}$, and if $-u_{1}$ belongs to $C$, the tangent cone to $K_{0}$ at $u_{0}$ (i.e. $C=\bar{\tau} \tau=0$

$$
\frac{\mathrm{d}^{+} u}{\mathrm{~d} t}(0)=2 P_{C} u_{1}-u_{1}
$$

where $P_{C}$ is the projection onto $C$.

\section{Proof. Part (a)}

1. Preliminary estimates

We have the energy equality for $\left(P_{\lambda}\right)$

$$
\frac{1}{2}\left|\dot{u}_{\lambda}(t)\right|^{2}+\phi_{\lambda}\left(u_{\lambda}(t)\right)=\frac{1}{2}\left|u_{1}\right|^{2}+\phi_{\lambda}\left(u_{0}\right)+\int_{0}^{t}\left(\dot{u}_{\lambda}(s), f(s)\right) \mathrm{d} s
$$


from which follows the inequality

$$
\begin{aligned}
& \frac{1}{2}\left|\dot{u}_{\lambda}(t)\right|^{2} \leqslant \frac{1}{2}\left|u_{1}\right|^{2}+\phi\left(u_{0}\right)+|f|_{L^{2}(0, T ; H)}\left(\int_{0}^{t}\left|\dot{u}_{\lambda}(s)\right|^{2} \mathrm{~d} s\right)^{t} \\
& \leqslant \frac{1}{2}\left|u_{1}\right|^{2}+\phi\left(u_{0}\right)+\frac{1}{2}|f|_{L^{2}(0, T ; H)}\left(1+\int_{0}^{t}\left|\dot{u}_{\lambda}(s)\right|^{2} \mathrm{~d} s\right)
\end{aligned}
$$

by the relation $x \leqslant\left(1+x^{2}\right) / 2$.

Gronwall's lemma implies:

$$
\phi_{\lambda}\left(u_{\lambda}(t)\right)+\left|\frac{1}{2} \dot{u}_{\lambda}(t)\right|^{2} \leqslant\left(\frac{1}{2}\left|u_{1}\right|^{2}+\phi\left(u_{0}\right)+\frac{1}{2}|f|_{L^{2}(0, T ; H)}\right) \exp \left(T|f|_{L^{2}(0, T ; H)}\right)
$$

Set

$$
E^{2} / 2=\left(\frac{1}{2}\left|u_{1}\right|^{2}+\phi\left(u_{0}\right)+\frac{1}{2}|f|_{L^{2}(0, T ; H)}\right) \exp \left(T|f|_{L^{2}(0, T ; H)}\right)
$$

We may therefore extract a subsequence, still denoted by $u_{k}$, such that

$$
\begin{aligned}
& u_{\lambda} \rightarrow u \text { in } C^{0}([0, T] ; H) \text { strong } \\
& \frac{\mathrm{d} u_{\lambda}}{\mathrm{d} t} \rightarrow \frac{\mathrm{d} u}{\mathrm{~d} t} \text { in } L^{\infty}(0, T ; H) \text { weak* } \\
& \phi_{\lambda}\left(u_{\lambda}\right) \rightarrow \chi \text { in } L^{\infty}(0, T) \text { weak }^{*} .
\end{aligned}
$$

As $\phi_{\lambda}\left(u_{\lambda}\right) \leqslant E,\left|u_{\lambda}-J_{\lambda} u_{\lambda}\right| \leqslant \sqrt{2 \lambda E}$ and $\phi\left(J_{\lambda} u_{\lambda}\right) \leqslant E$. We have

$$
E \geqslant \lim _{\lambda \rightarrow 0} \phi\left(J_{\lambda} u_{\lambda}(t)\right) \geqslant \phi(u(t))
$$

and $u(t) \in \operatorname{dom} \phi$, for all $t$ in $[0, T]$. Hence we obtain (1) and (2).

2. Main estimate

Let $a$ be the center of a closed ball of radius $\rho$, contained in the interior of dom $\phi$. By general theorems, we may suppose that $\phi$ is bounded on $a+B_{\rho}$ by a constant $C$. For an arbitrary continuous $z$ such that $|z(t)| \leqslant 1$ for all $t$ in $[0, T]$, we may write

$$
\left(\partial \phi_{\lambda}(u(t)), a+z(t)-u(t)\right) \leqslant \phi_{\lambda}(a+\rho z(t))-\phi_{\lambda}\left(u_{\lambda}(t)\right) .
$$

Integrating from 0 to $T$, we have

$$
\begin{aligned}
& \rho \int_{0}^{T}\left(\partial \phi_{\lambda}\left(u_{\lambda}(t)\right), z(t)\right) \mathrm{d} t \leqslant C T+\int_{0}^{T}\left(\frac{\mathrm{d}^{2} u_{\lambda}}{\mathrm{d} t^{2}}-f, a-u_{\lambda}\right) \mathrm{d} t \leqslant \\
& \quad \leqslant C T+\left.\left(\frac{\mathrm{d} u_{\lambda}}{\mathrm{d} t}, a-u_{\lambda}\right)\right|_{0} ^{T}+\int_{0}^{T}\left|\frac{\mathrm{d} u_{\lambda}}{\mathrm{d} t}\right|^{2} \mathrm{~d} t+\int_{0}^{T}\left(f, a-u_{\lambda}\right) \mathrm{d} t \\
& \leqslant C T+E\left(2|a|+2\left|u_{0}\right|+T E\right)+T E^{2}+|f|_{L^{2}(0, T ; H)} \sqrt{T}\left(|a|+\left|u_{0}\right|+T E\right) .
\end{aligned}
$$

If we choose

$$
z(t)=\frac{1}{\rho} \frac{\partial \phi_{\lambda}\left(u_{\lambda}(t)\right)}{\left|\partial \phi_{\lambda}\left(u_{\lambda}(t)\right)\right|}
$$

we obtain the estimate 


$$
\int_{0}^{T}\left|\partial \phi_{\lambda}\left(u_{\lambda}(t)\right)\right| \mathrm{d} t \leqslant C^{\prime} \text { independent of } \lambda
$$

We may therefore extract a new subsequence, still denoted by $u_{\lambda}$, such that $\partial \phi_{\lambda}\left(u_{\lambda}\right) \rightarrow \mu$ vaguely in $M^{1}([0, T] ; H)$, the set of bounded measures on $[0, T]$ with values in $H$. In the sense of distributions, $\mathrm{d}^{2} u / \mathrm{d} t^{2}+\mu=f$, which is precisely (3).

If $v$ is an arbitrary element of $C^{0}([0, T] ; H)$,

$$
\lim _{\lambda \rightarrow 0} \phi_{\lambda}(v(t))=\phi(v(t)) \text {. }
$$

Let $v$ be such that $\phi(v) \in L^{1}(0, T)$. Then $\phi\left(v_{\lambda}\right)$ is also in $L^{1}(0, T)$. We have

$$
\int_{0}^{T}\left(\phi_{\lambda}(v(t))-\phi_{\lambda}\left(u_{\lambda}(t)\right)\right) \mathrm{d} t \geqslant \int_{0}^{T}\left(\partial \phi_{\lambda}\left(u_{\lambda}(t)\right), v(t)-u_{\lambda}(t)\right) \mathrm{d} t .
$$

It is clear that the right-hand side tends to

$$
\langle\mu, v-u\rangle
$$

as $\lambda$ goes to infinity.

We have

$$
\lim \int_{0}^{T} \phi_{\lambda}\left(u_{\lambda}(t)\right) \geqslant \int_{0}^{T} \phi_{\lambda}\left(u_{\lambda}(t)\right) \mathrm{d} t \leqslant \int_{0}^{T} \phi(u(t)) \mathrm{d} t .
$$

Hence, taking the upper limit in (12), we get

$$
\int_{0}^{T}(\phi(v(t))-\phi(u(t))) d t \geqslant\langle\mu, v-u\rangle, \text { i.e. (4) }
$$

Interpretation of $\mu$.

Let $\mathrm{d} \mu=g \cdot \mathrm{d} t+\mathrm{d} \mu_{s}$ be the decomposition of $\mu$ with respect to the Lebesgue measure on $[0, T]$. Then Corollary 5.A of ref. [5], gives us the following results:

$$
g(t) \in \partial \phi(u(t)) \text { almost everywhere (with respect to the Lebesgue measure) on }[0, T] \text {. }
$$

Let $N(t)$ be the normal cone to $K_{0}=\overline{\operatorname{dom} \phi}$ at $u(t)$. Let $\mu_{s}=h .\left|\mu_{s}\right|$ with $h$ a $\mu_{s}$-integrable function. Then

$$
h(t) \in N(t) \quad\left|\mu_{s}\right|-\text { almost everywhere on }[0, T] .
$$

We shall often say in what follows that $\mu$ is the measure associated with the solution $u$ of $(P)$.

3. The properties of $\mathrm{d} u / \mathrm{d} t$

We know that the injection $M^{1}(0, T) \subsetneq H^{-1}(0, T)$ is compact. As $\mu_{\lambda}$ converges vaguely to $\mu$ in $M^{1}(0, T ; H) \cong\left(M^{1}(0, T)\right)^{N}, \mu_{\lambda}$ converges strongly to $\mu$ in $H^{-1}(0, T ; H) \cong\left(H^{-1}(0, T)\right)^{N}$, and therefore

$$
\frac{\mathrm{d} u_{\lambda}}{\mathrm{d} t} \rightarrow \frac{\mathrm{d} u}{\mathrm{~d} t} \text { strongly in } L^{2}(0, T ; H) .
$$

As $\left|\mathrm{d} u_{\lambda} / \mathrm{d} t\right| \leqslant E$, for all $t$ and $\lambda,\left(\mathrm{d} u_{\lambda} / \mathrm{d} t\right) \rightarrow(\mathrm{d} u / \mathrm{d} t)$ in $L^{p}(0, T ; H)$, for all $p \in[1, \infty)$. On the other hand, as $\mathrm{d}^{2} u / \mathrm{d} t^{2}$ is a measure, $\mathrm{d} u / \mathrm{d} t$ has right and left limits in every point of $(0, T)$, a right limit in 0 , and a left limit in $T$, and we can write 
4. Energy conservation

$$
\begin{aligned}
& \frac{\mathrm{d}^{-} u}{\mathrm{~d} t}=u_{1}+\int_{0}^{T} f(s) \mathrm{d} s-\mu([0, T)) \\
& \frac{\mathrm{d}^{+} u}{\mathrm{~d} t}=u_{1}+\int_{0}^{T} f(s) \mathrm{d} s-\mu([0, T]) .
\end{aligned}
$$

It is clear that

$$
\lim _{\lambda \rightarrow 0} \int_{0}^{T} \frac{\left|u_{\lambda}-J_{\lambda} u_{\lambda}\right|^{2}}{\lambda} \mathrm{d} t=0
$$

because $u_{\lambda}-J_{\lambda} u_{\lambda} \rightarrow 0$ in $C^{0}([0, T] ; H)$ and $\left(u_{\lambda}-J_{\lambda} u_{\lambda}\right) / \lambda \rightarrow \mu$ in $M^{1}([0, T] ; H)$. On the other hand, $\left(\left|u_{\lambda}-J_{\lambda} u_{\lambda}\right|^{2}\right) / \lambda$ is bounded in $L^{\infty}(0, T)$. Thus, $\left(\left|u_{\lambda}-J_{\lambda} u_{\lambda}\right|^{2}\right) / \lambda$ converges to 0 in $L^{\infty}(0, T)$ weak*. As $\phi_{\lambda}\left(u_{\lambda}\right)$ converges to a certain $\chi, \phi\left(J_{\lambda} u_{\lambda}\right)-\phi_{\lambda}\left(u_{\lambda}\right)-\left|u_{\lambda}+J_{\lambda} u_{\lambda}\right|^{2} /(2 \lambda)$ has the same limit in $L^{\infty}(0, T)$ weak*. We have the inequality

$$
\int_{0}^{T}\left(\phi(u)-\phi\left(J_{\lambda} u_{\lambda}\right)\right) \mathrm{d} t \geqslant\left\langle\mu_{\lambda}, u-J_{\lambda} u_{\lambda}\right\rangle \quad \text { as } \mu_{\lambda}(t) \in \partial \phi\left(J_{\lambda} u_{\lambda}(t)\right) \text { for } t \in[0, T] .
$$

Passing to the limit :

$$
\int_{0}^{T}(\phi(u)-\chi) d t \geqslant 0
$$

But :

$$
\lim \phi\left(J_{\lambda} u_{\lambda}\right) \geqslant \phi(u) .
$$

This implies $\chi \geqslant \phi(u)$, thus proving that $\chi=\phi(u)$.

If $\mu\left(\left\{t_{0}\right\}\right)=0$, we see from (9) that

hence:

$$
\lim _{\lambda \rightarrow 0} \phi_{\lambda}\left(u_{\lambda}\left(t_{0}\right)\right)=\lim _{\lambda \rightarrow 0} \phi\left(u_{\lambda}\left(t_{0}\right)\right)=\frac{1}{2}\left|u_{1}\right|^{2}+\phi\left(u_{0}\right)+\int_{0}^{t}(\dot{u}(s) f(s)) \mathrm{d} s
$$

$$
\frac{1}{2}|\dot{u}(t)|^{2}+\phi(u(t))=\frac{1}{2}\left|u_{1}\right|^{2}+\phi\left(u_{0}\right)+\int_{0}^{t}(\dot{u}(s), f(s)) \mathrm{d} s, \text { almost everywhere on }[0, T] .
$$

5. Initial conditions

It is quite clear that $u(0)=u_{0}$.

To finish the proof, we need only consider the interpretation (13), (14) of $\mu$. In fact, $N(t)=\partial \psi_{K_{0}}(u(t))$. If $\mathrm{d}^{+} u / \mathrm{d} t(0) \neq u_{1}$, then $\mu_{s}=\left(-u_{1}+\mathrm{d}^{+} u / \mathrm{d} t(0)\right) \delta_{0}+\tilde{\mu}_{s}$, and the conclusion of Part (a) of Theorem 1 holds.

Part (b)

The case when $u_{0} \in$ int $K_{0}$ is quite simple. So we make the hypothesis $u_{0} \in \partial K_{0}$. The idea of the proof is to compare $u_{\lambda}$ with the solution $v_{\lambda}$ of

$$
\left\{\begin{array}{l}
\frac{\mathrm{d}^{2} v_{\lambda}}{\mathrm{d} t^{2}}+\left(\partial \psi_{c}\right)_{\lambda}\left(v_{\lambda}-u_{0}\right)=0 \\
v_{\lambda}(0)=u_{0} \\
\frac{\mathrm{d} v_{\lambda}}{\mathrm{d} t}=u_{1}
\end{array}\right.
$$


Notice that $\partial \psi_{c \lambda}(x)=\left(x-P_{C} x\right) / \lambda$; $v_{\lambda}$ is given explicitly by

$$
\begin{array}{ll}
v_{\lambda}(t)=u_{0}+t P_{C} u_{1}+\sqrt{\lambda} \sin (t / \sqrt{\lambda})\left(u_{1}-P_{C} u_{1}\right) & \text { if } 0 \leqslant t \leqslant \pi \sqrt{\lambda} \\
v_{\lambda}(t)=u_{0}+t P_{C} u_{1}+(t-\pi \sqrt{\lambda})\left(u_{1}-P_{C} u_{1}\right) & \text { if } t \geqslant \pi \sqrt{\lambda}
\end{array}
$$

We can check these formulas using the fact that the decomposition of an arbitrary element of $H$ into the sum of its projections onto $C$ and $C^{\perp}=N$ is unique. This result is given in ref. [3], Lemma 2.2. Knowing that $\left(\partial \psi_{C}\right)_{\lambda}$ is Lipschitzian, we can see that $v_{\lambda}$ is the solution of (15). Let us show now that $\partial \phi_{\lambda}$ is locally near $\left(\partial \psi_{c}\right)_{\lambda}$ in an adequate sense.

Proposition 1. If $\phi$ is Lipschitzian in a neighbourhood of $u_{0}$, there exists positive $\rho$ and $\lambda_{0}$ such that, for $\left|x-u_{0}\right| \leqslant \rho$ and $\lambda \leqslant \lambda_{0}$ :

$$
\left(\partial \psi_{c}\right)_{\lambda}(x)-\partial \phi_{\lambda}(x)=h\left(x-u_{0}\right) / \hat{\lambda}+A_{\lambda}(x)
$$

where: $h\left(x-u_{0}\right)=P_{K_{0}}(x)-P_{c}\left(x-u_{0}\right)$;

$|h(x)| \leqslant|x| \varepsilon(|x|)$ and $\varepsilon(r)$ decreases to zero as $r$ goes to zero;

$h(x)=0$ on $K_{0}-u_{0}$

$\left|A_{\lambda}(x)\right| \leqslant k$

Proof. 1. Let $\phi$ be Lipschitzian over $\operatorname{dom} \phi \cap \overline{B_{\sigma}\left(u_{0}\right)}$ with Lipschitz constant $k$. Then dom $\phi \cap \overline{B_{\sigma}\left(u_{0}\right)}$ is closed: in fact, if $\left(x_{n}\right)_{n}$, is a sequence of points of $\operatorname{dom} \phi \cap \overline{B_{\sigma}\left(u_{0}\right)}$ converging to $x_{\infty}$, we have

$$
\phi(x) \leqslant \lim _{n \rightarrow \infty} \phi\left(x_{n}\right) \leqslant \lim _{n \rightarrow \infty}\left(k\left|x_{n}-x_{p}\right|+\phi\left(x_{p}\right)\right)=\phi\left(x_{p}\right)+\left|x-x_{p}\right|,
$$

which shows that $x \in \operatorname{dom} \phi$.

2. Lipschitzian extension of $\left.\phi\right|_{\overline{B_{\sigma}\left(u_{0}\right)}}$.

Denote by $\tilde{\phi}$ the convex function equal to $\phi$ in dom $\phi \cup \overline{B_{\sigma}\left(u_{0}\right)}$ and $+\infty$ elsewhere. Obviously $\tilde{\phi}$ is lower semicontinuous.

Define a convex function $\chi$ by $\chi(x)=\sup \{\tilde{\phi}(y)+(z, x-y): y \in$ int $\operatorname{dom} \tilde{\phi}$ and $z \in \partial \tilde{\phi}(y)\}$.

In fact $\chi$ is a Lipschitzian extension of $\left.\tilde{\phi}\right|_{\operatorname{dom} \tilde{\phi}}: \chi(x)$ is never infinite, thanks to the relation

$$
\chi(x) \geqslant \tilde{\phi}(y)+k|x-y|
$$

(clearly $\partial \tilde{\phi}$ is bounded by $k$ on int dom $\tilde{\phi}$ ).

If $x \in \operatorname{dom} \tilde{\phi}, \chi(x) \geqslant \tilde{\phi}(x)$. On the other hand, given $\varepsilon>0$, there exists a $y_{\varepsilon} \in \operatorname{int} \operatorname{dom} \tilde{\phi}$ and a $z_{\varepsilon} \in \partial \tilde{\phi}\left(y_{\varepsilon}\right)$ such that

$$
\chi(x) \leqslant \tilde{\phi}\left(y_{\varepsilon}\right)+\left(z_{\varepsilon}, x-y_{\varepsilon}\right)+\varepsilon \text {. }
$$

Therefore

$$
\chi(x) \leqslant \tilde{\phi}(x)+\varepsilon
$$

As $\varepsilon$ is arbitrary, we obtain $\chi(x)=\tilde{\phi}(x)$. We can extend this equality to all of the domain of $\tilde{\phi}$, by continuity. Let us show that $\chi$ is Lipschitzian: let $x$ and $x^{\prime}$ be given in $H$; we have

$$
\begin{aligned}
\chi\left(x^{\prime}\right)-\chi(x) & \leqslant \tilde{\phi}\left(y_{\varepsilon}^{\prime}\right)+\left(z_{\varepsilon}^{\prime}, x^{\prime}-y_{\varepsilon}^{\prime}\right)+\varepsilon-\tilde{\phi}\left(y_{\varepsilon}^{\prime}, x-y_{\varepsilon}^{\prime}\right) \\
& =\left(z_{\varepsilon}^{\prime}, x^{\prime}-x\right)+\varepsilon \leqslant k\left|x^{\prime}-x\right|+\varepsilon,
\end{aligned}
$$


if $\varepsilon$ is arbitrary and $y_{\varepsilon}^{\prime}$ and $z_{\varepsilon}^{\prime}$ are chosen adequately. We can bound from below $\chi\left(x^{\prime}\right)-\chi(x)$ by the same type of argument, and thus we have shown that $\left|\chi\left(x^{\prime}\right)-\chi(x)\right| \leqslant k\left|x^{\prime}+x\right|$.

3. Decomposition of $\bar{\phi}$.

Denote by $\psi$ the indicator function of $\operatorname{dom} \tilde{\phi}=\tilde{K}$.

Obviously $\tilde{\phi}=\psi+\chi$; then by Theorem 23.8 of ref. [2], $\partial \tilde{\phi}(x)=\partial \chi(x)+\partial \psi(x)$ for all $x$ in $\tilde{K}$. We have

$$
\partial \tilde{\phi}_{\lambda}(x)=\left(x-(I+\lambda \partial \tilde{\phi})^{-1} x\right) / \lambda
$$

Let $(I+\lambda \partial \tilde{\phi})^{-1} x=z_{\lambda}$ :

$$
z_{\lambda}+\lambda \partial \psi\left(z_{\lambda}\right)+\lambda g_{\lambda} \ni x
$$

where $g_{\lambda} \in \partial \chi\left(z_{\lambda}\right)$ and is thus bounded by $k$;

$$
\begin{aligned}
z_{\lambda} & =(I+\lambda \partial \psi)^{-1}\left(x-\lambda g_{\lambda}\right) . \\
\left|\partial \delta_{\lambda}(x)-\partial \psi_{\lambda}(x)\right| & =\left|\left[x-(I+\lambda \partial \psi)^{-1}\left(x-\lambda g_{\lambda}\right)-x+(I+\lambda \partial \psi)^{-1} x\right]\right| / \lambda \\
& =1 / \lambda\left|(I+\lambda \partial \psi)^{-1}\left(x-\lambda g_{\lambda}\right)-(I+\lambda \partial \psi)^{-1} x\right| \leqslant\left|g_{\lambda}\right| \leqslant k
\end{aligned}
$$

Lemma 4.6. of Zarantonello [3] allows us to write: $P_{R}\left(x+u_{0}\right)=u_{0}+P_{C} x+h(x)$ with

$$
|h(x)| \leqslant|x| \varepsilon(|x|) \quad \text { and } h(x)=0 \text { if } x \in \tilde{K}-u_{0} \text {. }
$$

Then

$$
\left|\partial \psi_{\lambda}(x)-\left(\partial \psi_{c}\right)_{\lambda}\left(x-u_{0}\right)\right|=\left|P_{C}\left(x-u_{0}\right)-P_{R^{R}}\left(x-u_{0}\right)\right| / \lambda \leqslant \varepsilon\left(\mid x-u_{0}\right)\left|x-u_{0}\right|
$$

4. Comparison of $\partial \phi_{\lambda}$ and $\partial \tilde{\phi}_{\lambda}$

The sequence $(I+\lambda \partial \phi)^{-1}$ converges to $P_{K_{0}} x$ when $\lambda$ goes to zero; but $(I+\lambda \partial \phi)^{-1}$ is a contraction; this convergence is therefore uniform on compact sets. So, there exists a function $j$ of $\rho$ and $\lambda$, decreasing to zero as $\lambda \rightarrow 0$, such that $\left|(I+\lambda \partial \phi)^{-1} x-P_{K_{0}} x\right| \leqslant j(\lambda, \rho)$ on the ball $B_{\rho}\left(u_{0}\right)$, and in an analogous fashion

$$
\left|(I+\lambda \partial \tilde{\phi})^{-1} x-P_{\tilde{K}} x\right| \leqslant \tilde{j}(\lambda, \rho) \quad \text { on the ball } B_{\rho}\left(u_{0}\right)
$$

Clearly, $P_{K_{0}}\left(B_{\rho}\left(u_{0}\right)\right) \subset B_{\rho}\left(u_{0}\right) \quad \forall \rho>0$

$$
P_{\hat{K}}\left(B_{\rho}\left(u_{0}\right)\right) \subset B_{\rho}\left(u_{0}\right) \quad \forall \rho>0 .
$$

Take $\rho=\sigma / 2$, and choose $\lambda_{0}$ so small that

$$
\max \left(j\left(\lambda_{0}, \sigma / 2\right), \tilde{J}\left(\lambda_{0}, \sigma / 2\right)\right)<\sigma / 2
$$

Then $\left|(I+\lambda \partial \phi)^{-1} x-u_{0}\right|<\sigma$, and $\left|(I+\lambda \partial \tilde{\phi})^{-1} x-u_{0}\right|<\sigma$ if $|x|<\sigma / 2$. Let $y=(I+\lambda \partial \phi)^{-1} x$ and $\tilde{y}=(I+\lambda \partial \delta)^{-1} x$. We know that $\left.\phi\right|_{B_{\sigma}\left(u_{0}\right)}=\left.\tilde{\phi}\right|_{B_{\sigma}\left(u_{0}\right)}$.

Therefore

$$
\begin{array}{ll}
(1 / \lambda)(x-y, v)+\phi(y+v)-\phi(y) \geqslant 0 & \forall v \in H \\
(1 / \lambda)(x-\tilde{y}, \tilde{v})+\tilde{\phi}(\tilde{y}+\tilde{v})-\tilde{\phi}(\tilde{y}) \geqslant 0 & \forall \tilde{v} \in H .
\end{array}
$$

If we add these two inequalities, taking $v=\tilde{y}-y$, and $\tilde{v}=-v$, we obtain $\tilde{y}=y$. This achieves the proof of Proposition 1. 
LEMMA 1. $u_{\lambda}$ and $v_{\lambda}$ satisfy the inequalities:

$$
\begin{aligned}
& \left|u_{\lambda}(t)-v_{\lambda}(t)\right| \leqslant c h \frac{t}{\sqrt{\lambda}}\left(k \frac{t^{2}}{2}+E \frac{t^{3}}{3 ! \lambda} \varepsilon(E t)\right) \\
& \left|\dot{u}_{\lambda}(t)-\dot{v}_{\lambda}(t)\right| \leqslant k t+\frac{E t^{2}}{2 \lambda} \varepsilon(E t)+\frac{1}{\lambda} \operatorname{ch} \frac{t}{\sqrt{\lambda}}\left(k \frac{t^{3}}{3 !}+\frac{E t^{4}}{4 ! \lambda} \varepsilon(E t)\right) .
\end{aligned}
$$

Proof. Define a transformation $T_{\lambda}$ on $C^{\circ}([O, T] ; H)$ by

$$
\left(T_{\lambda} w\right)(t)=u_{0}+t u_{1}+\int_{0}^{t}\left(f(s)-\partial \phi_{\lambda}(w(s))\right)(t-s) \mathrm{d} s .
$$

Then $u_{\lambda}$ satisfies

$$
T_{\lambda} u_{\lambda}=u_{\lambda}
$$

Estimate $T_{\lambda}^{n} w-T_{\lambda}^{n} \hat{w}$ by a standard recurrence argument:

$$
\mid\left(T_{\lambda} w-T_{\lambda}(\hat{w})(t) \mid \leqslant \frac{1}{\lambda} \frac{t^{2}}{2}\|w-\hat{w}\|_{C^{o}\left(\left[0, t_{0}\right]\right)} \quad \text { if } t \leqslant t_{0} .\right.
$$

Then

$$
\left|\left(T_{\lambda}^{n} w-T_{\lambda}^{n} \hat{w}\right)(t)\right| \leqslant \frac{t^{2 n}}{(2 n) ! \lambda^{n}}\|w-\hat{w}\|_{c^{\mathrm{o}}\left(\left[0, t_{0}\right]\right)} \quad \text { if } t \leqslant t_{0}
$$

As we know that $T_{\lambda}^{n} w$ converges to $u_{\lambda}$ as $n \rightarrow \infty$, for any initial $w$, and on any compact time interval, we may write

$$
\left|u_{\lambda}(t)-w(t)\right| \leqslant \sum_{n \geqslant 0}\left\|T_{\lambda}^{n+1} w-T_{\lambda}^{n} w\right\|_{C^{\circ}\left(\left[0, t_{0}\right]\right)} \leqslant c h \frac{t}{\sqrt{\lambda}}\left\|T_{\lambda} w-w\right\|_{C^{\circ}\left[\left[0 . t_{0}\right]\right)} \quad \text { if } t \leqslant t_{0} .
$$

Take $w=v_{\lambda}$ defined by (15).

$$
\left(T_{\lambda} v_{\lambda}-v_{\lambda}\right)(t)=u_{0}+t u_{1}-\int_{0}^{\lambda} \partial \phi_{\lambda}\left(v_{\lambda}(s)\right)(t-s) \mathrm{d} s-u_{0}-t u_{1}+\int_{0}^{t}\left(\partial \psi_{c}\right)_{\lambda}\left(v_{\lambda}(s)\right)(t-s) \mathrm{d} s .
$$

If we assume $t_{0} \leqslant \rho / E$ and $\lambda \leqslant \lambda_{0}$, we may apply the conclusions of Proposition 1 for $x=v_{\lambda}(t)$. Therefore

$$
\left|\left(T_{\lambda} v_{\lambda}-v_{\lambda}\right)(t)\right| \leqslant \int_{0}^{t}\left(k+\frac{E s \varepsilon(E s)}{\lambda}\right)(t-s) \mathrm{d} s \leqslant k \frac{t_{0}^{2}}{2 !}+E \frac{t_{0}^{3}}{3 ! \lambda} \varepsilon\left(E t_{0}\right) \quad \text { if } t \leqslant t_{0},
$$

and finally we obtain the estimate (17).

The estimate (18) is a straightforward consequence of (17), thanks to the relation

$$
\left|\dot{u}_{\lambda}(t)-\dot{v}_{\lambda}(t)\right| \leqslant \int_{0}^{\lambda} \mid\left(\partial \psi_{c}\right)_{\lambda}\left(v_{\lambda}(t)\right)-\partial \phi_{\lambda}\left(v_{\lambda}(t)\left|\mathrm{d} t+\int_{0}^{\lambda}\right| \partial \phi_{\lambda}\left(v_{\lambda}(t)\right)-\partial \phi_{\lambda}\left(u_{\lambda}(t)\right) \mid \mathrm{d} t .\right.
$$

End of proof of Theorem 1, part b.

Denote $\tilde{u}_{1}=2 P_{C} u_{1}-u_{1}$. Clearly, $\tilde{u}_{1}$ is an element of $C$. Let us notice that, for any given $\eta>0$, there exists a $\delta(\eta)>0$ such that, if $\left|P_{K_{0}} v-u_{0}\right| \leqslant \delta(\eta)$, then

$$
\left(v-P_{K_{0}} v, \tilde{u}_{1}\right) \leqslant \eta\left|\tilde{u}_{1}\right|\left|v-P_{K_{0}} v\right| \text {. }
$$


If it were not the case, we could find a sequence $v_{n}$ with $P v_{n}$ converging to $u_{0}$, and a strictly positive number $\eta_{0}$ such that

$$
\left(v_{n}-P_{K_{0}} v_{n}, \tilde{u}_{1}\right) \geqslant \eta_{0}\left|\tilde{u}_{1}\right|\left|v_{n}-P_{K_{0}} v_{n}\right|
$$

We can see that $\left(v_{n}+P v_{n}\right) /\left|v_{n}+P v_{n}\right|$ converges to a certain $w$, which must be in $N$, and therefore, we obtain a contradiction. We may assume $\eta(\delta)$ to be an increasing function of $\delta$, such that

$$
\left|P_{K_{0}} v-u_{0}\right| \leqslant \delta \text { implies }\left(v-P_{K_{0}} v, \tilde{u}_{1}\right) \leqslant \eta(\delta)\left|\tilde{u}_{1}\right|
$$

Write now

$$
\begin{gathered}
\left(u_{\lambda}(t), \tilde{u}_{1}\right)=\left(u_{\lambda}(\pi \sqrt{\lambda}), \tilde{u}_{1}\right)+\left(\dot{u}_{\lambda}(\pi \sqrt{\lambda}), \tilde{u}_{1}\right)(t-\pi \sqrt{\lambda})+\left(\int_{\pi \sqrt{\lambda}}^{t}\left(f(s)-A_{\lambda} u_{\lambda}(s)\right)(t-s) \mathrm{d} s, \tilde{u}_{1}\right) \\
\quad-\int_{\pi \sqrt{\lambda}}^{t}\left(\left[u_{\lambda}(s)-P_{K_{0}} u_{\lambda}(s)\right], \tilde{u}_{1}\right)(t-s) \mathrm{d} s \\
\geqslant\left(u_{\lambda}(\pi \sqrt{\lambda}), \tilde{u}_{1}\right)+\left(\dot{u}_{\lambda}(\pi \sqrt{\lambda}), \tilde{u}_{1}\right)(t+\pi \sqrt{\lambda}) \\
+|f|_{L^{2}} \frac{t^{3 / 2}}{\sqrt{\lambda})}+k \frac{t^{2}}{2}-\eta(E t) \int_{\pi \sqrt{\lambda}}^{t}(1 / \lambda)\left|u_{\lambda}(s)-P_{K_{0}} u_{\lambda}(s)\right|\left|\tilde{u}_{1}\right|(t-s) \mathrm{ds}
\end{gathered}
$$

From Proposition 1, and the estimate (11), we have:

$$
\int_{0}^{T}(1 / \lambda)\left|u_{\lambda}(s)-P_{K_{0}} u_{\lambda}(s)\right| \mathrm{d} s \leqslant C^{\prime}+k T .
$$

We obtain, in the limit as $\lambda \rightarrow 0$

$$
\begin{gathered}
\left(u(t)-u_{0}, \tilde{u}_{1}\right) \geqslant\left|\tilde{u}_{1}\right|^{2} t-o(t), \text { from where } \\
\left(\frac{\mathrm{d}^{+} u}{\mathrm{~d} t}(0), \tilde{u}_{1}\right) \geqslant\left|\tilde{u}_{1}\right|^{2} .
\end{gathered}
$$

On the other hand, as a result of (6), and of the fact that $\phi$ is continuous in a neighbourhood of $u_{0}$

$$
\left|\frac{\mathrm{d}^{+} u}{\mathrm{~d} t}(0)\right|=\left|u_{1}\right|=\left|\tilde{u}_{1}\right| . \quad \text { Conclusion (8) is now clear. }
$$

This completes the proof of Theorem 1 .

\section{3.a Discontinuity}

\section{COUNTEREXAMPLES TO WELLPOSEDNESS}

The idea of this counterexample is quite simple.

Take $H=\mathbf{R}^{2}, K=\left\{\left(x_{1}, x_{2}\right): x_{1} \geqslant 0, x_{1}+x_{2} \geqslant 0\right\}, \phi=\psi_{k}, f=0$; take as initial data $u_{0}^{h}=\left(1-h, \frac{1}{2}+h\right), u_{1}^{h}=\left(-1,-\frac{1}{2}\right)$ where $|h|<\frac{1}{2}$. It is easy to check that the solutions are unique.

If $h=0$, according to part (b) of Theorem 1 , the solution is

$$
\begin{array}{ll}
u^{0}(t)=\left(1-t, \frac{1}{2}(1-t)\right) & \text { if } t \leqslant 1 \\
u^{0}(t)=\left(\frac{1}{2}(t-1), t-1\right) & \text { if } t>1
\end{array}
$$


If $h>0$, the solution is

$$
u^{h}(t)=\left(1-h-t, \frac{1}{2}+h-\frac{1}{2} t\right) \quad \text { if } t \leqslant 1
$$

and it has a unique reflection at time $t=1$ on the side $x_{1}+x_{2}=0$ of $K$. Then

$$
u^{h}(t)=\left(\frac{1}{2}(t-1)-h, t-1+h\right) \quad \text { if } t>1 .
$$

If $h<0$, the solution has a first reflection at time $t=1+2 h$; then $u^{h}(t)=(-3 h-(t-1-2 h)$, $(t-1-2 h) / 2)$ until the time $1+4 h$, when it has a second reflection, after which

$$
u^{h}(t)=\left(-\frac{1}{2} t+\frac{1}{2}-h, t-1+h\right) .
$$

As $h$ tends to zero, remaining negative, $u^{h}$ tends to $\tilde{u}^{0}$ defined by

$$
\begin{aligned}
& \tilde{u}^{0}(t)=\left(1+t, \frac{1}{2}(1-t)\right) \quad \text { if } t \leqslant 1 \\
& \tilde{u}_{0}(t)=\left(-\frac{1}{2}(t-1), t-1\right) \quad \text { if } t>1 .
\end{aligned}
$$

We can see now that, as a function of $h, u^{h}$ is right- but not left-continuous.

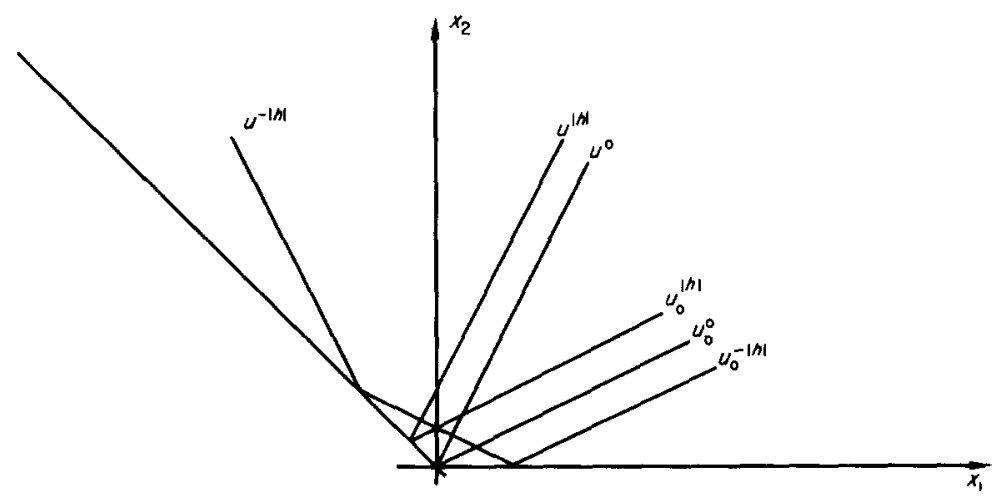

Fig. 2.

\section{3. b. Nonuniqueness}

Two examples will be given, both of them with $\phi=\psi_{K}$.

(i). Take $H=\mathbf{R}, K=\mathbf{R}^{+}, \phi=\psi_{K}, u_{0}=u_{1}=0$.

We seek an infinitely differentiable nonpositive $f$ which gives us two solutions. The first solution will be formed of an infinite number of arches, smaller and smaller as $t$ gets nearer to 0 (see figure below). The associated measure $\mu$ will then be atomic. The second solution will be $u=0, \mu=f$.

More precisely, let $\rho \in \mathscr{D}(\mathbf{R})$ be an even, nonnegative function, the support of which is included in $[-1,+1]$, and satisfying $\int_{R} \rho(x) \mathrm{d} x=1$. Denote $\rho_{\varepsilon}(x)=\rho(x / \varepsilon)$. Let $y_{\varepsilon}=1_{[\varepsilon / 2,1-\varepsilon / 2]} * \rho_{\varepsilon / 2}$ with $\varepsilon<\frac{1}{2}$. Clearly $y_{\varepsilon}=1$ on $[\varepsilon, 1-\varepsilon]$, supp $y_{\varepsilon} \subset[0,1]$, and $y_{\varepsilon}$ is $C^{\infty}$. It is an easy exercise to check that

$$
2 \int_{0}^{1} y_{\varepsilon}(s)(1-s) \mathrm{d} s-\int_{0}^{1} y_{\varepsilon}(s) \mathrm{d} s=0 .
$$




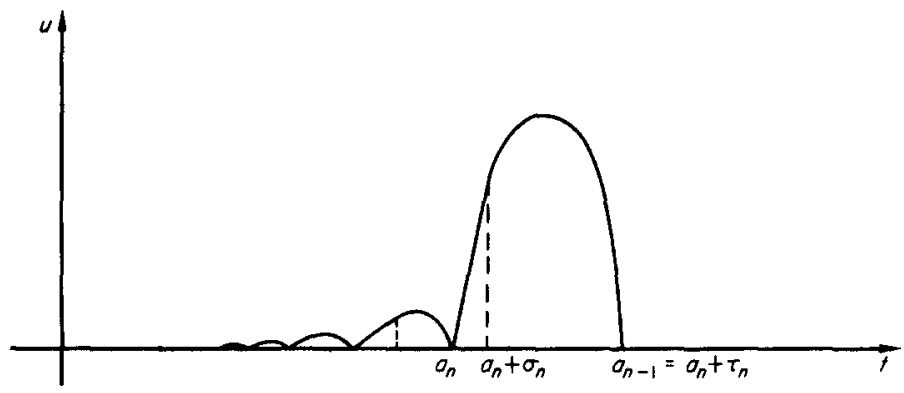

Fig. 3.

From now on we fix an $\varepsilon$, and write $y_{\varepsilon}=y, y_{1}=\int_{0}^{1} y(s) \mathrm{d} s$ and $y_{2}=\int_{0}^{1} y(s)(1+s) \mathrm{d} s$. Obviously $y_{1}=2 y_{2}$. We look for an $f$ defined on each interval $\left[a_{n}, a_{n-1}\right]$ as follows: $f=0$ on $\left[a_{n}, a_{n}+\sigma_{n}\right]$

$$
f(t)=-\eta_{n} y\left(\frac{t-a_{n}-\sigma_{n}}{\tau_{n}-\sigma_{n}}\right) \text { on }\left[a_{n}+\sigma_{n}, a_{n}+\tau_{n}\right]=\left[a_{n}+\sigma_{n}, a_{n-1}\right] .
$$

$f$ is $C^{\infty}$ except perhaps in 0 , if it is defined.

We have necessarily

$$
\begin{aligned}
& \ddot{u}=f \quad \text { on }\left[a_{n}, a_{n-1}\right] \\
& u\left(a_{n}\right)=u\left(a_{n-1}\right)=0
\end{aligned}
$$

If we write $\frac{\mathrm{d}^{+} u}{\mathrm{~d} t}\left(a_{n}\right)=v_{n}$ then $\frac{\mathrm{d}^{-} u}{\mathrm{~d} t}\left(a_{n-1}\right)=-v_{n-1}$.

Our aim is now to look for necessary conditions which will insure that $f$ has a meaning and is $C^{\infty}$, and $u$ has a meaning and is a solution of $(\mathrm{P})$.

$$
\begin{array}{ll}
u(t)=v_{n}\left(t-a_{n}\right) & \text { on }\left[a_{n}, a_{n}+\sigma_{n}\right] \\
u(t)=v_{n}\left(t-a_{n}\right)-\eta_{n} \int_{a_{n}+a_{n}}^{t} y(s)(t+s) \mathrm{d} s & \text { on }\left[a_{n}+\sigma_{n}, a_{n-1}\right],
\end{array}
$$

which gives us

$$
\begin{aligned}
v_{n} \tau_{n} & =\eta_{n} y_{2}\left(\tau_{n}-\sigma_{n}\right)^{2} \\
v_{n}+v_{n-1} & =\eta_{n} y_{1}\left(\tau_{n}-\sigma_{n}\right) .
\end{aligned}
$$

Let $v_{n-1}=\alpha_{n} v_{n}$. Then

$$
\tau_{n}=\sigma_{n} \frac{\alpha_{n}+1}{\alpha_{n}-1} \text { and } \eta_{n} \sigma_{n}=\frac{v_{n}\left(\alpha_{n}^{2}-1\right)}{2 y_{1}} \text {. }
$$

Choose $\alpha_{n}=n$ for $n \geqslant 4$. This implies

$$
\eta_{n} \sigma_{n}=\frac{v_{4}}{2 y_{1}} \frac{\left(n^{2}-1\right) 4 !}{n !}=\frac{12 v_{4}}{y_{1}} \frac{n^{2}-1}{n !} .
$$


Take

$$
\eta_{n}=12 \frac{v_{4}}{y_{1}} \frac{1}{(n-4) !} \text { and } \sigma_{n}=\frac{n+1}{n(n-2)(n-3)} .
$$

For $u$ to be a solution of $(\mathrm{P})$, we have only to check that

$$
\begin{aligned}
& \sum_{n \geqslant 4} \mu\left(\left\{a_{n}\right\}\right)=2 \sum_{n \geqslant 4} v_{n}=2 \sum_{n \geqslant 4} \frac{1}{n !} 24 v_{4}<+\infty \\
& \sum_{n \geqslant 4} \tau_{n}=\sum_{n \geqslant 4} \frac{n+1}{n(n-2)(n-3)} \frac{n+1}{n-1}<+\infty .
\end{aligned}
$$

Then $a_{4}<+\infty$ and we can choose $T=a_{4}$.

For $f$ to be $C^{\infty}$ up to zero we need

$$
\eta_{n} /\left(\sum_{m \geqslant n-1} \tau_{n}\right)^{r} \sim \frac{1}{(n-4) !}\left(C_{n}\right)^{p} \frac{v_{1}}{2 y_{1}}<+\infty \quad \forall p ;
$$

thus, we obtain the supplementary information that $f^{(p)}(0)=0, \forall p \in \mathbf{N}$. Clearly 0 with associated measure $\hat{\mu}=f$ is a solution. Thus we have built an infinitely differentiable $f$ such that (P) has two solutions.

(ii) Take $H=\mathbf{R}^{2}, f=0$. We intend to build a convex set $K$ of $C^{\infty}$ boundary, such that with an initial velocity tangent to the boundary, we obtain two different solutions; one "along" the boundary, the other having an infinity of reflexions on it.

We shall use the results and notations of the preceding paragraph. Let us define a continuous parametrized curve with values in $\mathbf{C} \simeq \mathbf{R}^{2}$ as follows:

$$
F(t)=F\left(a_{n}\right)+\mathrm{e}^{i \beta_{n}}\left(t-a_{n}\right)+\mathrm{e}^{i\left(\beta_{n}-\pi / 2\right)} u(t) \quad \text { if } t \in\left[a_{n}, a_{n-1}\right] 0 \leqslant t \leqslant T=a_{4} .
$$

This curve will be continuously differentiable if

$$
\mathrm{e}^{i \beta_{n}}\left(1-i \frac{\mathrm{d}^{+} u}{\mathrm{~d} t}\left(a_{n}\right)\right)=\mathrm{e}^{i \beta_{n+1}}\left(1-i \frac{\mathrm{d}^{-} u}{\mathrm{~d} t}\left(a_{n}\right)\right) .
$$

Then, necessarily $\beta_{n}-\beta_{n+1}=2 \arctan v_{n}$. As $v_{n}=24 v_{4} / n$ !, the series $\sum \beta_{n}$ converges. Moreover, if we choose $v_{4}$ small enough, $\sum_{n=4} \beta_{n}<\left\langle\pi / 2\right.$, which means that $t \mapsto{ }^{n} F(t)$ will have no tangent parallel to the imaginary axis. We have $F^{\prime \prime}(t)=\mathrm{e}^{i\left(\beta_{n}-\pi / 2\right)} f(t)$ on $\left[a_{n}, a_{n-1}\right]$. But $f$ is constructed in such a way that $f^{(p)}\left(a_{n}\right)=0$ for all $p \geqslant 0, n \geqslant 4$. Therefore $F$ is $C^{\infty}$ on $\left.[0, T]\right]^{p}$ Moreover, the sign of the curvature of $F([0, T])$ is the sign of $\dot{F}_{1} \ddot{F}_{2}-\dot{F}_{2} \ddot{F}_{1}$, where $F=F_{1}+i F_{2}$.

We can easily check that $\dot{F}_{1} \ddot{F}_{2}-\dot{F}_{2} \ddot{F}_{1}=-f(t) \geqslant 0$. We can thus see that $F([0, T])$ is the boundary of a convex set $K$ of $\mathbf{C} \simeq \mathbf{R}^{2}$.

Take now initial conditions $u_{0}=0, \dot{u}_{0}=a>U$, and define a function $\psi_{a}$ on $[0, T]$ by

$$
\psi_{a}(t)=\frac{1}{a} \int_{0}^{t}|\dot{F}(\sigma)| \mathrm{d} \sigma
$$

Let $\phi_{a}$ be the reciprocal of $\psi_{a}$.

Then $v(t)=F\left(\phi_{a}(t)\right)$ is a solution of $(P)$ on $\left[0, \psi_{a}(T)\right]$.

In fact, $v(t) \in K$, for all $t \in\left[0, \psi_{a}(T)\right]$. 


$$
\begin{aligned}
\frac{\mathrm{d} v}{\mathrm{~d} t} & =a \dot{F}\left(\phi_{a}(t)\right) /\left|\dot{F}\left(\phi_{a}(t)\right)\right| \\
\frac{\mathrm{d}^{2} v}{\mathrm{~d} t^{2}} & =a \frac{\mathrm{d}}{\mathrm{d} t}\left[\dot{F}\left(\phi_{a}(t)\right) /\left|\dot{F}\left(\phi_{a}(t)\right)\right|\right] .
\end{aligned}
$$

As $\dot{F}(\sigma) \neq 0$, for all $\sigma \in[0, T], \mathrm{d}^{2} u / \mathrm{d} t^{2}$ is a bounded function which can be identified with a bounded measure. On the other hand, it is clear that $\mathrm{d}^{2} v / \mathrm{d} t^{2}$ is normal to $\partial K,(\mathrm{~d} v / \mathrm{d} t)$ is continuous at every point, $|\mathrm{d} v / \mathrm{d} t|=a$ and the initial conditions are satisfied. Therefore $u$ is a solution of $(\mathrm{P})$ in the sense of Definition 1.

The other solution will be given by

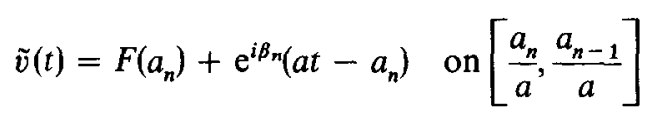

Clearly, $\tilde{v}(t) \in K$ for all $t$. We have

$$
\begin{aligned}
& \frac{\mathrm{d}^{+} \tilde{v}}{\mathrm{~d} t}\left(a_{n} / a\right)=a \mathrm{e}^{i \beta_{n}} \\
& \frac{\mathrm{d}^{-} \tilde{v}}{\mathrm{~d} t}\left(a_{n} / a\right)=a \mathrm{e}^{i \beta n+1} \\
& \frac{\mathrm{d}^{2} \tilde{v}}{\mathrm{~d} t^{2}}=0 \quad \text { if } t \in\left(a_{n}, a_{n-1}\right) .
\end{aligned}
$$

Therefore

$$
\frac{\mathrm{d}^{2} \tilde{v}}{\mathrm{~d} t^{2}}=a \sum_{n \geqslant 1} \delta_{a_{n} / a}\left(\mathrm{e}^{i \beta_{n}}-\mathrm{e}^{i \beta_{n+1}}\right)=-\tilde{\mu} .
$$

It is easy to check, using the interpretation of the measure associated with a solution of (P), (13), (14), that $\tilde{v}$ is a solution of $(\mathrm{P})$; clearly, the energy is conserved, and the initial conditions are satisfied.

Comments on this part. These results are closely connected with results on the propagation of singularities of a hyperbolic problem on a manifold with boundary. The first one obviously refers to diffraction in an angle. The second one was figured out by thinking of a tennis player who succeeds in making his ball bounce higher and higher, from a rest position, only by hitting it downwards. Professor L. Amerio told me that he had built an analogous example. The third type of counterexample is given, with a different construction by M. E. Taylor in his paper [4] concerning reflection of singularities of wave equations in an exterior domain of $\mathbf{R}^{N}$. When the complement of this domain is not convex, Taylor's theorem fails as there appear phenomena of nonuniqueness of the wave front set. I cannot see, presently, how all these results could be taken in account together in reasonable mathematical theory.

\section{A UNIQUENESS THEOREM IN A PARTICULAR CASE}

THEOREM 2. Let $\phi=\psi_{K}, f=0$. Assume that the boundary $\partial K$ of the closed convex set $K$ is of class $C^{3}$, and that its gaussian curvature is strictly positive. Then the problem $(P)$ admits a unique solution on $[0,+\infty]$ in the sense of Definition 1. Moreover, if the initial data $u_{0}$ is given 
on $\partial K$ and $u_{1}$ is tangent to $\partial K$, then $u$ runs along the geodesic of $\partial K$ passing through $u_{0}$ and tangent to $u_{1}$ with the speed $\left|u_{1}\right|$. If the initial data are not such, then $u$ is never tangent to $\partial K$, and it has a finite number of reflections in a finite time.

Proof. Denote by $\mathscr{S}_{K}(u)$ the tangent cone to $K$ at $u$, i.e. $\mathscr{T}_{K}(u)=\overline{\cup_{s}>0} \tau(K-u)$ and by $n(u)$ the exterior unit normal to $K$ at $u$. Remark that

$$
\mathscr{S}_{K}(u)=\{v:(v, n(u)) \geqslant 0\} \quad \text { for all } u \in \partial K .
$$

Let us first prove the local uniqueness at every point $t$. If $u(t) \in$ int $K$, or if $u(t) \in \partial K$ and $\left(\mathrm{d}^{-} u / \mathrm{d} t\right)(t)$ is the interior of $\mathscr{S}_{K}(u(t))$, the local uniqueness is immediate.

If $u(t) \in \partial K$, and $\mathrm{d}^{-} u / \mathrm{d} t(t) \notin \mathscr{S}_{K}(u(t))$, then

$$
\left|\frac{\mathrm{d}^{+} u}{\mathrm{~d} t}(t)\right|=\left|\frac{\mathrm{d}^{-} u}{\mathrm{~d} t}(t)\right| .
$$

and

$$
\frac{\mathrm{d}^{+} u}{\mathrm{~d} t}(t)-\frac{\mathrm{d}^{-} u}{\mathrm{~d} t}(t)=k n(u(t))
$$

according to Theorem 1 .

Necessarily the vector

$$
\frac{\mathrm{d}^{+} u}{\mathrm{~d} t}(t)=\frac{\mathrm{d}^{-} u}{\mathrm{~d} t}(t)-2\left(\frac{\mathrm{d}^{-} u}{\mathrm{~d} t}(t), n(u(t))\right) n(u(t))
$$

is in the interior of $\mathscr{S}_{K}(u(t))$. The local uniqueness is then clear. The only difficult case is when $u(t) \in \partial K$, and $\left(\left(\mathrm{d}^{-} u / \mathrm{d} t(t)\right), n(u(t))\right)=0$. We need the following result.

LEMMA 2. If $\partial K$ is of class $C^{3}$ and its gaussian curvature is strictly positive, then there exists no sequence of points of $\partial K,\left(u_{m}\right)_{m \in N}$ converging to $u_{\infty}$, such that $u_{m}$ is a reflexion point for all $m$, i.e.

$$
\left\{\begin{array}{l}
\frac{\left(u_{m}-u_{m-1}, n\left(u_{m}\right)\right)}{\left|u_{m}-u_{m-1}\right|}=\frac{\left(u_{m}-u_{m+1}, n\left(u_{m}\right)\right)}{\left|u_{m}-u_{m+1}\right|} \text { and } \\
\frac{u_{m}-u_{m-1}}{\mid u_{m}-u_{m-1}}-\frac{\left(u_{m}-u_{m-1}, n\left(u_{m}\right)\right) n\left(u_{m}\right)}{\mid u_{m}-u_{m-1} 1}=\frac{u_{m+1}-u_{m}}{\left|u_{m+1}-u_{m}\right|}-\frac{\left(u_{m+1}-u_{m}, n\left(u_{m}\right)\right) n\left(u_{m}\right)}{\left|u_{m+1}-u_{m}\right|}
\end{array}\right.
$$

such that $u_{m+1}-u_{m}$ has a limit direction, and such that

$$
\sum_{m \in \mathbf{N}}\left|u_{m+1}-u_{m}\right|<+\infty \text {. }
$$

Let us show that this lemma implies local uniqueness. With $u_{0} \in \partial K,\left(n\left(u\left(t_{0}\right)\right),\left(\mathrm{d}^{-} u / \mathrm{d} t\right)\left(t_{0}\right)\right)=0$, then necessarily $\left(\left(d^{+} u / \mathrm{d} t\right)\left(t_{0}\right), n\left(u\left(t_{0}\right)\right)\right)=0$. Suppose that there is no right neighbourhood of $t_{0}$ such that $u(t)$ belongs to $\partial K$ in all this neighbourhood. Then we can find a $t_{1}$, arbitrarily near $t_{0}$, such that $u\left(t_{1}\right) \notin \partial K$. There must exist at most a finite number of reflections between $t_{0}$ and $t_{1}$, in order to satisfy Lemma 2 . Then $\left(\left(\mathrm{d}^{+} u / \mathrm{d} t\left(t_{0}\right)\right), n\left(u\left(t_{0}\right)\right)\right)<0$ and we obtain a contradiction. Therefore there exists an $\eta>0$ such that $u(t) \in \partial K$ for $t_{0} \leqslant t \leqslant t_{0}+\eta$. 
Let $\mu$ be the measure associated with $u$, and define a real valued measure $(\mu, z)$ with $z \in C^{0}([0, T] ; H)$ by

$$
\langle(\mu, z), \phi\rangle=\langle\mu, z \phi\rangle \quad \forall \phi \in C^{0}([0, T]) .
$$

Let $s=(w-(w, n(u)) n(u)) \chi$ with $w \in H$, and $\chi \in C^{0}([0, T])$, supp $\chi \subset\left[t_{0}, t_{0}+\eta\right]$. It is clear that $s(t) \in \mathscr{S}_{K}(u(t))$, and $s$ is universally integrable on $[0, T]$. According to the interpretation of $\mu,(13)$ and (14), necessarily $\langle\mu, s\rangle=0$.

So

$$
\left.(\mu, w)\right|_{\left(t_{0}, t_{0}+\eta\right)}=(n(u), w)\left(\left.\mu\right|_{\left(t_{0}, t_{0}+\eta\right)^{2}} n(u)\right) .
$$

If we set $v=\left(\left.\mu\right|_{\left(t_{0}, t_{0}+\eta\right)}, n(u)\right)$, we may then identify $\left.\mu\right|_{\left(t_{0}, t_{0}+\eta\right)}$ and $n(u) v$. Suppose $u\left(t_{0}\right)=0$, which does not restrict the generality, and denote $n\left(t_{0}\right)=n_{0}, u_{N}=-\left(u, n_{0}\right), u^{\prime}=u-\left(u, n_{0}\right) n_{0}$. Represent $\partial K$ in a neighbourhood of 0 as follows:

$$
|u| \leqslant \alpha \text { and } u_{N}=f\left(u^{\prime}\right) \text { imply } u \in \partial K .
$$

Here $f$ is convex, twice continuously differentiable, and $D f(0)=0$. We differentiate the relation $u_{N}(t)=f\left(u^{\prime}(t)\right)$ on $\left[t_{0}, t_{0}+\eta\right]$.

$$
\frac{\mathrm{d} u_{N}}{\mathrm{~d} t}=D f\left(u^{\prime}(t)\right) \frac{\mathrm{d} u^{\prime}}{\mathrm{d} t}
$$

$\frac{\mathrm{d} u}{\mathrm{~d} t}$ is continuous, as $\left|\frac{\mathrm{d}^{+} u}{\mathrm{~d} t}\right|=\left|\frac{\mathrm{d}^{-} u}{\mathrm{~d} t}\right|, \frac{\mathrm{d}^{+} u}{\mathrm{~d} t}-\frac{\mathrm{d}^{-} u}{\mathrm{~d} t}=k n(u)$ (for a certain real $k$ ), and

$$
\left(\frac{\mathrm{d}^{+} u}{\mathrm{~d} t}, n(u)\right)=\left(\frac{\mathrm{d}^{-} u}{\mathrm{~d} t}, n(u)\right)=0 .
$$

We now differentiate (19) in the sense of distributions:

$$
\frac{\mathrm{d}^{2} u_{N}}{\mathrm{~d} t^{2}}=D^{2} f\left(u^{\prime}(t)\right) \frac{\mathrm{d} u^{\prime}}{\mathrm{d} t} \frac{\mathrm{d} u^{\prime}}{\mathrm{d} t}+D f\left(u^{\prime}(t)\right) \frac{\mathrm{d}^{2} u^{\prime}}{\mathrm{d} t}
$$

Explicitly

$$
n(u)=\frac{\left(D f\left(u^{\prime}\right) ;-1\right)}{\left(1+\left|D f\left(u^{\prime}\right)\right|^{2}\right)^{1 / 2}}
$$

whence

$$
\begin{aligned}
& \frac{\mathrm{d}^{2} u^{\prime}}{\mathrm{d} t^{2}}=\frac{-v D f\left(u^{\prime}\right)}{\left(1+\left|D f\left(u^{\prime}\right)\right|^{2}\right)^{1 / 2}} \\
& \frac{\mathrm{d}^{2} u_{\mathrm{N}}}{\mathrm{d} t^{2}}=\frac{v}{\left(1+\left|D f\left(u^{\prime}\right)\right|^{2}\right)^{1 / 2}}, \\
& \left(D f\left(u^{\prime}\right), \frac{\mathrm{d}^{2} u^{\prime}}{\mathrm{d} t^{2}}\right)=-\frac{v\left|D f\left(u^{\prime}\right)\right|^{2}}{\left(1+\left|D f\left(u^{\prime}\right)\right|^{2}\right)^{1 / 2}}
\end{aligned}
$$

Substituting in relation (20) we obtain

$$
v\left(1+\left|D f\left(u^{\prime}\right)\right|^{2}\right)^{1 / 2}=D^{2} f\left(u^{\prime}\right) \frac{\mathrm{d} u^{\prime}}{\mathrm{d} t} \frac{\mathrm{d} u^{\prime}}{\mathrm{d} t} .
$$


Therefore, $v$ can be identified with a function and

$$
\frac{\mathrm{d}^{2} u}{\mathrm{~d} t^{2}}+\frac{D^{2} f\left(u^{\prime}\right) \frac{\mathrm{d} u^{\prime}}{\mathrm{d} t} \frac{\mathrm{d} u^{\prime}}{\mathrm{d} t}}{\left(1+\left|D f\left(u^{\prime}\right)\right|^{2}\right)^{1 / 2}} n(u)=0 .
$$

This is precisely the equation of the geodesics of $\partial K$. As $\partial K$ is of class $C^{3}$, we know there is uniqueness. By a classical argument, local uniqueness implies global uniqueness.

To complete the proof of Theorem 2, we need only to establish Lemma 2.

Proof of Lemma 2. Suppose there exists a sequence $u_{m} \in \partial K$ such that

(i) $u_{m} \rightarrow u_{\infty}$ as $m \rightarrow \infty$,

(ii) $\frac{Q_{m}\left(u_{m}-u_{m+1}\right)}{\left|u_{m}-u_{m+1}\right|}=\frac{Q_{m}\left(u_{m}-u_{m-1}\right)}{\left|u_{m-1}-u_{m}\right|}$,

(iii) $\frac{P_{m}\left(u_{m}-u_{m+1}\right)}{\left|u_{m}-u_{m+1}\right|}=\frac{P_{m}\left(u_{m-1}-u_{m}\right)}{\left|u_{m-1}-u_{m}\right|}$

where $Q_{m} x=-\left(x, n\left(u_{m}\right)\right), P_{m} x=x+n\left(u_{m}\right) Q_{m} x$.

In a neighbourhood $U$ of $u_{\infty}$ we have the representations of $\partial K$ given by

$$
u \in \partial K \cap U \Rightarrow Q_{m}\left(u-u_{m}\right)=f_{m}\left(P_{m}\left(u-u_{m}\right)\right)
$$

where $f_{m}$ is of class $C^{3}$ and $D f_{m}(0)=0 ; f_{m}$ is convex.

Obviously

$$
\frac{f_{m}\left(P_{m}\left(u_{m-1}-u_{m}\right)\right)}{\left|P_{m}\left(u_{m-1}-u_{m}\right)\right|}=\frac{f_{m}\left(P_{m}\left(u_{m+1}-u_{m}\right)\right)}{\left|P_{m}\left(u_{m+1}-u_{m}\right)\right|} .
$$

On the other hand,

$$
f_{m}\left(u^{\prime}\right)=D^{2} f_{m}(0) u^{\prime} u^{\prime}+\left|u^{\prime}\right|^{2} g_{m}\left(u^{\prime}\right) \quad \text { if }\left(u^{\prime}, n\left(u_{m}\right)\right)=0,
$$

with

$$
\left|g_{m}\left(u^{\prime}\right)\right| \leqslant h\left(\left|u^{\prime}\right|\right)
$$

The function $h$ does not depend on $m ; \lim _{r \rightarrow 0} h(r)=0$, and $h$ is increasing

$$
\text { Set } \begin{aligned}
\kappa_{m} & =D^{2} f_{m}(0)\left(\frac{P_{m}\left(u_{m-1}-u_{m}\right)}{\left|P_{m}\left(u_{m-1}-u_{m}\right)\right|}\right)\left(\frac{P_{m}\left(u_{m-1}-u_{m}\right)}{\mid P_{m}\left(u_{m-1}-u_{m} \mid\right.}\right) \\
& =D^{2} f_{m}(0)\left(\frac{P_{m}\left(u_{m+1}-u_{m}\right)}{\left|P_{m}\left(u_{m+1}-u_{m}\right)\right|}\right)\left(\frac{P_{m}\left(u_{m+1}-u_{m}\right)}{\left|P_{m}\left(u_{m+1}-u_{m}\right)\right|}\right)
\end{aligned}
$$

From (21) and (22) we obtain:

$$
\begin{aligned}
\kappa_{m}\left(\left|P_{m}\left(u_{m-1}-u_{m}\right)\right|\right. & \left.-\left|P_{m}\left(u_{m+1}-u_{m}\right)\right|\right)+\left|P_{m}\left(u_{m-1}-u_{m}\right)\right| g_{m}\left(P_{m}\left(u_{m-1}-u_{m}\right)\right) \\
& -\left|P_{m}\left(u_{m+1}-u_{m}\right)\right| g_{m}\left(P_{m}\left(u_{m+1}-u_{m}\right)\right)=0
\end{aligned}
$$

Since we have assumed that the gaussian curvature of $\partial K$ at $u_{\infty}$ is strictly positive, $\kappa_{m} \geqslant \kappa_{0}>0$ for $m$ large enough. Dividing both sides of (23) by $\left|P_{m}\left(u_{m-1}-u_{m}\right)\right|$ we obtain 


$$
\begin{aligned}
\kappa_{0}\left|\frac{\left|P_{m}\left(u_{m+1}-u_{m}\right)\right|}{\left|P_{m}\left(u_{m-1}-u_{m}\right)\right|}-1\right| \leqslant \mid & \left|\frac{\left|P_{m}\left(u_{m+1}-u_{m}\right)\right|}{\left|P_{m}\left(u_{m-1}-u_{m}\right)\right|}-1\right| h\left(\left|P_{m}\left(u_{m+1}-u_{m}\right)\right|\right) \\
& +h\left(\left|P_{m}\left(u_{m-1}-u_{m}\right)\right|\right)+h\left(\left|P_{m}\left(u_{m+1}-u_{m}\right)\right|\right) .
\end{aligned}
$$

If we choose $m_{0}$ such that

$$
m \geqslant m_{0} \Rightarrow h\left(\left|P_{m}\left(u_{m+1}-u_{m}\right)\right|\right) \leqslant \kappa_{0} / 2,
$$

then

$$
\frac{\kappa_{0}}{2}\left|\frac{\left|P_{m}\left(u_{m+1}-u_{m}\right)\right|}{\left|P_{m}\left(u_{m-1}-u_{m}\right)\right|}-1\right| \leqslant h\left(\left|P_{m}\left(u_{m-1}-u_{m}\right)\right|\right)+h\left(\left|P_{m}\left(u_{m+1}-u_{m}\right)\right|\right) \text {. }
$$

Set $l_{m}=\left|u_{m-1}-u_{m}\right|$. Finally

$$
\frac{\kappa_{0}}{2}\left|\frac{\left|P_{m}\left(u_{m+1}-u_{m}\right)\right|}{\left|P_{m}\left(u_{m-1}-u_{m}\right)\right|}-1\right| \leqslant h\left(l_{m}\right)+h\left(l_{m+1}\right) .
$$

On the other hand we have

$$
P_{m}\left(u_{m-1}-u_{m}\right)-f_{m}\left(P_{m}\left(u_{m-1}-u_{m}\right)\right) n\left(u_{m}\right)=u_{m-1}-u_{m} .
$$

Therefore

$$
\begin{aligned}
\left|l_{m}-\right| P_{m}\left(u_{m-1}-u_{m}\right)|| \leqslant & \left|D^{2} f_{m}(0)\left(P_{m}\left(u_{m-1}-u_{m}\right)\right)\left(P_{m}\left(u_{m-1}+u_{m}\right)\right)\right|+ \\
& +\left|P_{m}\left(u_{m-1}-u_{m}\right)\right|^{2} h\left(\left|P_{m}\left(u_{m-1}-u_{m}\right)\right|\right) .
\end{aligned}
$$

So we have a constant $K$ such that

$$
\left|\frac{l_{m}}{\left|P_{m}\left(u_{m-1}-u_{m}\right)\right|}-1\right| \leqslant K l_{m}
$$

and analogously

$$
\left|\frac{l_{m+1}}{\left|P_{m}\left(u_{m+1}-u_{m}\right)\right|}-1\right| \leqslant K l_{m+1} .
$$

From (24), (25) and (26) we obtain

$$
\left|l_{m+1} / l_{m}-1\right| \leqslant K^{\prime}\left(h\left(l_{m}\right)+h\left(l_{m+1}\right)+l_{m}+l_{m+1}\right) .
$$

Set $\tilde{h}(r)=K^{\prime}(h(r)+r)$, then

$$
l_{m+1} \geqslant l_{m}+l_{m}\left(\tilde{h}\left(l_{m+1}\right)+\tilde{h}\left(l_{m}\right)\right) .
$$

Since $l_{m}$ converges to 0 , we can find an arbitrarily large $m_{0}$ such that $l_{m} \leqslant l_{m_{0}}$, for all $m \geqslant m_{0}$. Then

$$
\begin{gathered}
l_{m+1} \geqslant l_{m}-2 l_{m} \tilde{h}\left(l_{m_{0}}\right) \quad \text { if } m \geqslant m_{0} . \\
\sum_{m \geqslant m_{0}+1} l_{m} \geqslant \sum_{m \geqslant m_{0}} l_{m}\left(1-2 \tilde{h}\left(l_{m_{0}}\right)\right)=\sum_{m \geqslant m_{0}+1}\left(1-2 \tilde{h}\left(l_{m_{0}}\right)\right) l_{m}+\left(1-2 \tilde{h}\left(l_{m_{0}}\right)\right) l_{m_{0}} .
\end{gathered}
$$

Therefore

$$
\sum_{m \geqslant m_{0}+1} l_{m} \geqslant \frac{1-2 \tilde{h}\left(l_{m_{0}}\right)}{2 \tilde{h}\left(l_{m_{0}}\right)} \cdot l_{m_{0}}
$$


It is clear that we can choose $m_{0}$ so large that the right hand side of this inequality is arbitrarily large. We obtain therefore a contradiction, as $u_{m+1}-u_{m}$ is supposed to have a limit direction when $m$ tends to infinity. This completes the proof of Theorem 2 .

The main results of this paper have been stated in ref. [6].

\section{REFERENCES}

1. Brezis H., Opérateurs Maximaux Monotones et Semi-Groupes de Contraction dans les Espaces de Hilbert. North Holland (1973).

2. Rockafellar R. T., Convex Analysis. Princeton Univ. Press (1970).

3. Zarantonello E. H., Contributions to Nonlinear Functional Analysis. pp. 237-424. Academic Press (1971).

4. TAYLOR M. E., Communs pure appl. Math. 38, 1-38 (1976).

5. Rockafellar R. T., Pacif. J. Math. 39, $439-469$ (1971).

6. Schatzman M., C.r. Acad. Sci. Paris 284, série A, 603-606 (1977). 